\title{
Genetics of congenital eye malformations: insights from chick experimental embryology
}

\author{
Paola Bovolenta ${ }^{1,2}$ and Juan-Ramón Martinez-Morales ${ }^{3}$ \\ ${ }^{1}$ Centro de Biología Molecular "Severo Ochoa," (CSIC/UAM) and ${ }^{2}$ CIBERER, ISCIII, \\ Madrid 28049, Spain \\ ${ }^{3}$ Centro Andaluz de Biología del Desarrollo (CSIC/UPO/JA), Seville 41013, Spain \\ \# Correspondence at pbovolenta@cbm.csic.es
}

Key words: tissue interaction; anopthalmia; microphthalmia; coloboma; optic cup; optic vesicle

\begin{abstract}
Embryological manipulations in chick embryos have been pivotal to understand many aspects of vertebrate eye formation. This research was particularly relevant to uncover the role of tissue interactions as drivers of eye morphogenesis and to dissect the function of critical genes. Here, we have highlight a few of these past experiments to argue how they can still help to search for yet undiscovered causes of rare congenital eye anomalies, such as microphthalmia anophthalmia and coloboma. We will also underscore a few similarities between the chicken and human eye that might be exploited to address degenerative ocular diseases.
\end{abstract}


Much of our current knowledge on how vertebrate embryos develop derives from studies $\mathrm{BC}$, Aristotele was the first to observe, describe and interpret the changes that an embryo undergoes inside its egg (Needham 1959). Since then, the chick embryo has been a valuable tool to establish basic developmental concepts such as, for example, tissue competence or to enable the discovery of key developmental factors, including NGF or Shh. Now, if the chick has been the organism of pick, the eye has been, among the possible systems/organs, the embryologists' frequent choice, which to focus on. In both cases, the reason is likely the same: the chick as a whole and the eye as an organ are easily accessible and amenable to experimental manipulations and culture conditions. It follows that classical embryological studies of chick eye development have set much of the basis of our current knowledge on how this organ develops and provided hints to understand the basis of human congenital eye malformations. Despite this important "historical" role, the chick embryo has currently lost some ground, at least as a model system for understanding eye diseases. This has been in favour of other species, such as the mouse or the zebrafish, in which genetic approaches are more amenable. Should we use more often the chick as a paradigm to study human genetic eye malformations and other ocular diseases?

There are a number of excellent recent reviews that answer this question and well advocate for the advantages (without forgetting the disadvantages) of the chick as a model to study eye development and its disorders. The reader is referred to them for more information (i.e. Vergara and Canto-Soler 2012; Wisely et al. 2017). In this perspective article, we will simply attempt to make the case that revisiting some of the past experiments in chick embryos may be useful to predict yet undiscovered causes of congenital eye malformations. To this end, we have selected a few examples picked among those studies that have linked eye malformations with relevant eye tissues' interactions, as we believe that the chick embryo has been a particularly amenable system to their understanding.

\section{Congenital eye anomalies}

The most common group of congenital eye malformations include microphthalmia (significant reduction of the globe axial length), anophthalmia (complete absence of the ocular globe) and coloboma (heterogeneous conditions characterized by failure mostly of ocular fissure closure but that can also affect the iris, eyelid, etc) or, as collectively known, MAC. MAC are interrelated and rare anomalies that can occur in an isolated form or associated with other malformations in complex syndromic conditions. They inevitably lead 
to severe visual deficits and can account for up to $11 \%$ of infant blindness in developed countries (Williamson and FitzPatrick 2014). MAC are generally caused by homozygous and heterozygous mutations, either hereditary or de novo, in very conserved eye developmental genes, although environmental factors have been also described (Williamson and FitzPatrick 2014). As yet, several candidate genes responsible for microphthalmia and anophthalmia have been identified. These are mostly transcription factors at the core of forebrain or lens gene regulatory networks (Beccari et al. 2013; Cvekl and Zhang 2017), such as SOX2, OTX2, VSX2/CHX10, RAX, FOXE3; key components of cell-to-cell communication, or genes involved in retinal progenitors' proliferation (Williamson and FitzPatrick 2014). The list of genes causative of human coloboma is also relatively large and depicts a "coloboma gene network", comprising transcription factors and signalling molecules largely related to ventral eye patterning (Gregory-Evans et al. 2004). Despite these notable advances, only part of the MAC patients receives accurate molecular diagnosis, pointing to the existence of possible alternative developmental mechanisms responsible of ocular malformation or of additional causative genes. The identification of these genes may come from the currently used genome sequencing approaches or searched among molecules involved in eye tissue interactions, the discovery of which was in large proportion favoured by experiments performed in chick embryos.

\section{Linking eye tissues' interaction in chick embryos with human eye malformations}

In all vertebrates, eye formation starts with the specification of the eye field within the anterior neural plate. In birds, as in mammals, the neural plate folds to form the neural tube; concomitantly, cells of the eye field splits and bulges out under the influence of the abutting axial meso-endoderm. The result is the formation of two lateral protrusions, known as optic vesicles. These vesicles are composed by a pseudostratified and apparently homogeneous neuroepithelium, which, upon thickening of the overlying ectoderm into the lens placode, begins to infold and generate two bi-layered optic cups (Hilfer 1983). Optic cup formation involves also the formation of a transient opening along the ventral retina, termed optic fissure, which will close after the ingression of the peri-ocular mesenchyme (POM) originating the retinal vasculature.

Thus, the eye forms thanks to the contribution of different tissues, the behaviour of which is coordinated by mutual signalling and inductive events (Figure 1). The developmental anomalies resulting from the failure of these tissues' cross-talk caught the researchers' 
attention already at the beginning of the twenty century, when chick eye tissues or the eye as a whole begun to be cultured or grafted in the flank of host embryos.

Retinal-POM interactions. Grafting approaches were further aided by the identification of natural chick mutants such as the "Creeper" that presents "phocomelic" limbs and microphthalmic and colobomatous eyes. By grafting Creeper or wild type (wt) donor optic vesicles into the flank of wt host embryo, Kenneth Gayer (Gayer 1942) asked if wt vesicles would acquire a Creeper phenotype and if Creeper vesicles, on the contrary, could ameliorate their phenotype. Indeed, he noted that wt vesicles, isolated from the surrounding tissue, the POM, developed coloboma together with sclera and RPE abnormalities; grafted Creeper vesicles instead improved their sclera defects but maintained the coloboma trait. Seen with our current knowledge, these results are quite fascinating because they provided the first evidence that retina-POM interaction is fundamental for fissure closure, as recently shown with similar grafting experiments in zebrafish (Gestri 2018). The recent sequence of the Creeper genome has revealed a mutation in the Indian hedgehog (Ihh) gene (Jin et al. 2016). Ihh, a member of the Hedgehog family of signalling proteins, is highly expressed in all mesenchyme, including the POM. Genetic inactivation of Ihh in mouse causes abnormalities in RPE pigmentation and mesenchymal condensation, the latter required to form the sclera (Dakubo et al. 2008). Thus, $I h h^{-/}$mice reproduce the ocular phenotype observed in the Creeper chick embryos, with the exception of the fissure coloboma. It follows that factors other than Ihh and expressed in the POM, but not in the flank mesenchyme, might be responsible of the Creeper eye coloboma. In zebrafish, knock-down of sox11 and sox4, two transcription factors of the SoxC subfamily expressed in the POM, results in ocular coloboma (Wen et al. 2015). Thus, searching for down-stream targets of Sox-C genes, using the easily dissectible chick POM, might aid the identification of additional causes of coloboma. Part of them might be linked to the control of Ihh signalling, given that, curiously, in zebrafish sox4 morphants ihh expression was found up-regulated and its abrogation counteracted the coloboma of sox4 morphants (Wen et al. 2015).

Retinal-Lens interactions. The apposition and growth coordination between the lens ectoderm and the optic vesicle neuroepithelium is another example of tissues' interaction that has been extensively studied in chick embryos (Coulombre and Coulombre 1964) and linked to abnormal eye development. Surgical elimination of the lens ectoderm before optic vesicle invagination, but not after optic cup formation, has been shown to prevent optic vesicle invagination (Hyer et al. 2003) and the proper segregation between neural retina and RPE (Hyer et al. 1998). This and other related experiments led to the long-standing idea that the 
two tissues mutually dependent for further development of the organ. Interepithelial filopodia mechanisms that have been proposed to mediate their cross talk (Chow and Lang 2001; see also, Adler and Canto-Soler 2007, for a critical review of the topic). The idea of retina-lens interdependence has been recently challenged by the observation that mammalian ES-cultured cells can generate self-organized eyes in the absence of a lens (Eiraku et al. 2011; Nakano et al. 2012) and that acquisition of a cup shape is an intrinsic property of the retinal neuroepithelium (Nicolas-Perez et al. 2016). Still, microphthamia of different phenotypic severity often results from mutations in genes expressed in the lens but not in the retina, such as FOXE3 (Williamson and FitzPatrick 2014), indicating that the lens influences retinal growth. The implantation of beads socked in given secreted factors - a technique particularly amenable in chick embryos- allowed the identification of a number of molecules, including members of the BMP, FGF and Wnt family, that support retinal growth in the absence of the lens ectoderm (rev in: Adler and Canto-Soler 2007). Yet, the specific contributions of members of the pathways activated by these signalling factors to microphthalmia and anophthalmia are still poorly explored, but might be worth further investigation. Indeed, mutations in SALL4 have been recently found in microphthalmic individuals (Ullah et al. 2017). SALL4, a transcription factor of the SAL family previously associated to a syndromic form of strabismus (the Okihiro/Duane-radial ray syndrome), is a down-stream effector of BMP signaling, pointing to the components of this complex pathway as potential candidates to explain unresolved cases of microphthalmia. It is also worth mentioning that many of the molecularly diagnosed cases of human anophthalmia and microphtalmia are due to mutations in genes such as SOX2, OTX2 or PAX6, which play multiple roles in the development of both the lens and the optic vesicle, as uncovered by several studies in chick embryos (see; Adler and Canto-Soler 2007, for a review). Thus, other factors expressed in both tissues might be good candidate genes. Among them, the transcription factor Meis1 seems an attractive possibility. Comparative transcriptomic analysis of wild type and Meisl mutant mice has shown that the expression of a large number molecules, linked to human microphthalmia and localized either to the lens, the retina, or both, depends on this transcription factor (Marcos et al. 2015).

Retinal-RPE interaction. The neural retina and the RPE derive from the same neuroepithelium of the optic vesicle but their genetic program becomes highly divergent as soon as the vesicle begins to infold. The result is the formation of two tissues with very different morphology, organization and properties. Studies in chick embryos have first 
suggested that the restriction of Otx2 expression to the presumptive RPE region is key to this divergence (Bovolenta et al. 1997), as thereafter confirmed with the use of Otx mouse mutants (Martinez-Morales et al. 2001). Subsequent studies have shown that RPE specification further depends on the additional activity of different isoforms of the Mitf transcription factor and of Wnt/ßcatenin signalling (Fuhrmann et al. 2014). Although Pax6 seems required to maintain RPE identity (Raviv et al. 2014), studies using birds have established that the combined activity of Wnt/ßcatenin and Otx2 (Westenskow et al. 2010), BMP and Wnt (Steinfeld et al. 2017), or Otx2 and Mitf independently (Martinez-Morales et al. 2003) suffices to convert neural retinal cells into retinal pigmented cells by activating the expression of genes of the melanogenic cascade. This shows that the retina is competent to acquire RPE characteristic, but the opposite is also true. For example, implantations of beads soaked in FGF ligands in proximity of the chick prospective RPE converts the tissue into an inverted neural retina, initiating its neurogenesis (Martinez-Morales et al. 2005; Vogel-Hopker et al. 2000). Notably in his early grafting experiments, Gayer (Gayer 1942) noted that wt grafted optic vesicle that developed coloboma were characterized by partial "retina duplications in the outer layer" with "an inverse arrangement of the retinal strata". Furthermore, failure in neural retina or RPE specification often culminates with the development of anophthalmia or microphthalmia, as shown by genetic manipulation of key transcription factors in different vertebrate species, including the chick (Tsukiji et al. 2009; Wang et al. 2016). Therefore additional genetic causes for MAC should be searched among the genes controlling the development of either one of the two tissues. The gene regulatory network controlling neural retinal development is fairly well understood, even in humans (Hoshino et al. 2017). In contrast, our knowledge of RPE specification seems limited, posing the question of whether Otx, Mitf, Wnt/ $\beta$ catenin and perhaps Pax6 are indeed sufficient for RPE specification. Embryological manipulations in chick embryos coupled with transcriptomic studies might help to give answer to this question.

\section{Linking chick eye studies with other ocular diseases}

The chicken eye differs from the human eye in several aspects but has also a number of similarities that makes it a suitable model to study ocular diseases other than MAC (Wisely et al. 2017). For example and in contrast to the mouse, the chick eye includes a true corneal Bowman's layer. This stratum is involved in corneal would healing, making the chick a good model to understand this event as well as to find treatment to improve it. Similar to the human, the chick eye has a stable blood-aqueous barrier and controls the aqueous humor 
through trabecular filtration (Wisely et al. 2017). Thus, the chick seems as a proper model to study ocular pressure and related disease, including glaucoma, a leading cause of blindness worldwide. Moreover, chickens and humans are diurnal animals and therefore their retinas are enriched in the type of photoreceptors that mediate light vision: the cones. Cone photoreceptors are particularly enriched in the fovea, a central spot in our retina that allows high-acuity vision. Although the chick retina has no proper fovea, it contains a cone-enriched high-acuity area. A very recent study has shown that the specification of this area depends on a spatially restricted high $F g f 8$ expression and the absence of retinoic acid (RA) activity controlled by its degrading enzymes. Notably, reduction of Fgf8 expression or manipulation of RA activity changes all the properties of the high-acuity area, including the specification of cone photoreceptors (da Silva and Cepko 2017). Given the highly conserved distribution of Fgf8 and RA regulatory enzymes in the human embryonic retina (da Silva and Cepko 2017), these data open new avenues to fight against photoreceptor degeneration. Indeed, photoreceptor degeneration is a common feature of retinitis pigmentosa, age-related macular degeneration and other retinal dystrophies that collectively affects millions of people worldwide, still with little effective cure (Letelier et al. 2017). Preventing cone death will be therefore a substantial step forward for many patients (Bovolenta and Cisneros 2009) and further studies on the chick high-acuity area might be critical in this respect.

\section{Conclusions}

Embryological manipulations in the chick embryos led the way for many years of the research aimed at understanding how the vertebrate eye forms. This research uncovered important signalling interactions among tissues and dissected the function of critical genes. With our current knowledge, many of these studies should be seen as an invaluable source of inspiration to search for new and yet poorly studied aspects of vertebrate eye development. The result might be the identification of unexpected players of eye development and, perhaps, of additional causes of genetic eye malformations. The recent identification of the receptor for the cleaved and active form of the complement component $\mathrm{C} 3$ (C3a) as a regulator of patterning, proliferation and survival of optic cup cells could be an example (GrajalesEsquivel et al. 2017). Notably, neutralization of C3aR function with specific antibodies causes microphthalmia and coloboma in treated chick embryos (Grajales-Esquivel et al. 2017).

Looking back to go forward could be an approach, but certainly not the only one. The specific characteristics of the chick eye, its experimental advantages and the increasing 
successful use of editing techniques to modify the chick genome (Gandhi et al. 2017; Williams et al. 2018) are calling for additional use of the chick as a useful and efficient model to address eye diseases.

Acknowledgements: Work in our labs is supported by the following grants: MINECO BFU2016-75412-R (including FEDER funds); PCIN-2015-176-C02-01/ERA-Net NeuronII and the CIBERER, ISCIII to PB; MINECO BFU2017-86339P and FEDER-2017 to JRMM; MINECO BFU2016-81887-REDT and Fundación Ramón Areces-2016 to both JRMM and PB and an Institutional Grant to the CBMSO from the Fundación Ramón Areces and Banco Santander.

\section{Conflict of interest statement}

On behalf of all authors, the corresponding author states that there is no conflict of interest.

\section{References}

Adler R, Canto-Soler MV (2007) Molecular mechanisms of optic vesicle development: complexities, ambiguities and controversies. Dev Biol 305: 1-13. doi: 10.1016/j.ydbio.2007.01.045

Beccari L, Marco-Ferreres R, Bovolenta P (2013) The logic of gene regulatory networks in early vertebrate forebrain patterning. Mech Dev 130: 95-111. doi: 10.1016/j.mod.2012.10.004

S0925-4773(12)00110-4 [pii]

Bovolenta P, Cisneros E (2009) Retinitis pigmentosa: cone photoreceptors starving to death. Nat Neurosci 12: 5-6. doi: 10.1038/nn0109-5

Bovolenta P, Mallamaci A, Briata P, Corte G, Boncinelli E (1997) Implication of OTX2 in pigment epithelium determination and neural retina differentiation. J Neurosci 17: 4243-52.

Chow RL, Lang RA (2001) Early eye development in vertebrates. Annu Rev Cell Dev Biol 17: 25596. doi: 10.1146/annurev.cellbio.17.1.255

Coulombre AJ, Coulombre JL (1964) Lens Development. I. Role of the Lens in Eye Growth. J Exp Zool 156: 39-47.

Cvekl A, Zhang X (2017) Signaling and Gene Regulatory Networks in Mammalian Lens Development. Trends Genet 33: 677-702. doi: 10.1016/j.tig.2017.08.001

da Silva S, Cepko CL (2017) Fgf8 Expression and Degradation of Retinoic Acid Are Required for Patterning a High-Acuity Area in the Retina. Dev Cell 42: 68-81 e6. doi: 10.1016/j.devcel.2017.05.024 
Dakubo GD, Mazerolle C, Furimsky M, Yu C, St-Jacques B, McMahon AP, Wallace VA (2008) Indian hedgehog signaling from endothelial cells is required for sclera and retinal pigment epithelium development in the mouse eye. Dev Biol 320: 242-55. doi: 10.1016/j.ydbio.2008.05.528

Eiraku M, Takata N, Ishibashi H, Kawada M, Sakakura E, Okuda S, Sekiguchi K, Adachi T, Sasai Y (2011) Self-organizing optic-cup morphogenesis in three-dimensional culture. Nature 472: 51-6. doi: 10.1038/nature09941

Fuhrmann S, Zou C, Levine EM (2014) Retinal pigment epithelium development, plasticity, and tissue homeostasis. Exp Eye Res 123: 141-50. doi: 10.1016/j.exer.2013.09.003

Gandhi S, Piacentino ML, Vieceli FM, Bronner ME (2017) Optimization of CRISPR/Cas9 genome editing for loss-of-function in the early chick embryo. Dev Biol 432: 86-97. doi: 10.1016/j.ydbio.2017.08.036

Gayer K (1942) A study of coloboma and other abnormalities in transplants of eye primordia from normal and Creeper chick embryos. Journal of Experimental Zoology 89: 103-133. doi: 10.1002/jez.1400890105

Gestri, G.; Bazin-Lopez, N.; Scholes, C.; Wilson, SW. (2018) Cell behaviours during closure of the choroid fissure in the developing eye. Frontiers in Cellular Neuroscience.

Grajales-Esquivel E, Luz-Madrigal A, Bierly J, Haynes T, Reis ES, Han Z, Gutierrez C, McKinney Z, Tzekou A, Lambris JD, Tsonis PA, Del Rio-Tsonis K (2017) Complement component C3aR constitutes a novel regulator for chick eye morphogenesis. Dev Biol 428: 88-100. doi: 10.1016/j.ydbio.2017.05.019

Gregory-Evans CY, Williams MJ, Halford S, Gregory-Evans K (2004) Ocular coloboma: a reassessment in the age of molecular neuroscience. J Med Genet 41: 881-91. doi: 10.1136/jmg.2004.025494

Hilfer SR (1983) Development of the eye of the chick embryo. Scan Electron Microsc: 1353-69.

Hoshino A, Ratnapriya R, Brooks MJ, Chaitankar V, Wilken MS, Zhang C, Starostik MR, Gieser L, La Torre A, Nishio M, Bates O, Walton A, Bermingham-McDonogh O, Glass IA, Wong ROL, Swaroop A, Reh TA (2017) Molecular Anatomy of the Developing Human Retina. Dev Cell 43: 763-779 e4. doi: 10.1016/j.devcel.2017.10.029

Hyer J, Kuhlman J, Afif E, Mikawa T (2003) Optic cup morphogenesis requires pre-lens ectoderm but not lens differentiation. Dev Biol 259: 351-63.

Hyer J, Mima T, Mikawa T (1998) FGF1 patterns the optic vesicle by directing the placement of the neural retina domain. Development 125: 869-77.

Jin S, Zhu F, Wang Y, Yi G, Li J, Lian L, Zheng J, Xu G, Jiao R, Gong Y, Hou Z, Yang N (2016) Deletion of Indian hedgehog gene causes dominant semi-lethal Creeper trait in chicken. Sci Rep 6: 30172. doi: 10.1038/srep30172

Letelier J, Bovolenta P, Martinez-Morales JR (2017) The pigmented epithelium, a bright partner against photoreceptor degeneration. J Neurogenet 31: 203-215. doi: 10.1080/01677063.2017.1395876

Marcos S, Gonzalez-Lazaro M, Beccari L, Carramolino L, Martin-Bermejo MJ, Amarie O, MateosSan Martin D, Torroja C, Bogdanovic O, Doohan R, Puk O, Hrabe de Angelis M, Graw J, GomezSkarmeta JL, Casares F, Torres M, Bovolenta P (2015) Meis1 coordinates a network of genes 
implicated in eye development and microphthalmia. Development 142: 3009-20. doi: 10.1242/dev.122176

Martinez-Morales JR, Del Bene F, Nica G, Hammerschmidt M, Bovolenta P, Wittbrodt J (2005) Differentiation of the vertebrate retina is coordinated by an FGF signaling center. Dev Cell 8: 56574. doi: 10.1016/j.devcel.2005.01.022

Martinez-Morales JR, Dolez V, Rodrigo I, Zaccarini R, Leconte L, Bovolenta P, Saule S (2003) OTX2 activates the molecular network underlying retina pigment epithelium differentiation. J Biol Chem 278: 21721-31. doi: 10.1074/jbc.M301708200

Martinez-Morales JR, Signore M, Acampora D, Simeone A, Bovolenta P (2001) Otx genes are required for tissue specification in the developing eye. Development 128: 2019-30.

Nakano T, Ando S, Takata N, Kawada M, Muguruma K, Sekiguchi K, Saito K, Yonemura S, Eiraku M, Sasai Y (2012) Self-formation of optic cups and storable stratified neural retina from human ESCs. Cell Stem Cell 10: 771-85. doi: 10.1016/j.stem.2012.05.009

Needham J (1959) A history of embryology.

Nicolas-Perez M, Kuchling F, Letelier J, Polvillo R, Wittbrodt J, Martinez-Morales JR (2016) Analysis of cellular behavior and cytoskeletal dynamics reveal a constriction mechanism driving optic cup morphogenesis. Elife 5. doi: 10.7554/eLife.15797

Raviv S, Bharti K, Rencus-Lazar S, Cohen-Tayar Y, Schyr R, Evantal N, Meshorer E, Zilberberg A, Idelson M, Reubinoff B, Grebe R, Rosin-Arbesfeld R, Lauderdale J, Lutty G, Arnheiter H, AsheryPadan R (2014) PAX6 regulates melanogenesis in the retinal pigmented epithelium through feedforward regulatory interactions with MITF. PLoS Genet 10: e1004360. doi: 10.1371/journal.pgen.1004360

Steinfeld J, Steinfeld I, Bausch A, Coronato N, Hampel ML, Depner H, Layer PG, Vogel-Hopker A (2017) BMP-induced reprogramming of the neural retina into retinal pigment epithelium requires Wnt signalling. Biol Open 6: 979-992. doi: 10.1242/bio.018739

Tsukiji N, Nishihara D, Yajima I, Takeda K, Shibahara S, Yamamoto H (2009) Mitf functions as an in ovo regulator for cell differentiation and proliferation during development of the chick RPE. Dev Biol 326: 335-46. doi: 10.1016/j.ydbio.2008.11.029

Ullah E, Wu D, Madireddy L, Lao R, Ling-Fung Tang P, Wan E, Bardakjian T, Kopinsky S, Kwok PY, Schneider A, Baranzini S, Ansar M, Slavotinek A (2017) Two missense mutations in SALL4 in a patient with microphthalmia, coloboma, and optic nerve hypoplasia. Ophthalmic Genet 38: 371-375. doi: 10.1080/13816810.2016.1217550

Vergara MN, Canto-Soler MV (2012) Rediscovering the chick embryo as a model to study retinal development. Neural Dev 7: 22. doi: 10.1186/1749-8104-7-22

Vogel-Hopker A, Momose T, Rohrer H, Yasuda K, Ishihara L, Rapaport DH (2000) Multiple functions of fibroblast growth factor-8 (FGF-8) in chick eye development. Mech Dev 94: 25-36.

Wang Z, Yasugi S, Ishii Y (2016) Chx10 functions as a regulator of molecular pathways controlling the regional identity in the primordial retina. Dev Biol 413: 104-11. doi: 10.1016/j.ydbio.2016.03.023

Wen W, Pillai-Kastoori L, Wilson SG, Morris AC (2015) Sox4 regulates choroid fissure closure by limiting Hedgehog signaling during ocular morphogenesis. Dev Biol 399: 139-53. doi: 10.1016/j.ydbio.2014.12.026 
Westenskow PD, McKean JB, Kubo F, Nakagawa S, Fuhrmann S (2010) Ectopic Mitf in the embryonic chick retina by co-transfection of beta-catenin and Otx2. Invest Ophthalmol Vis Sci 51: 5328-35. doi: 10.1167/iovs.09-5015

Williams RM, Senanayake U, Artibani M, Taylor G, Wells D, Ahmed AA, Sauka-Spengler T (2018) Genome and epigenome engineering CRISPR toolkit for in vivo modulation of cis-regulatory interactions and gene expression in the chicken embryo. Development. doi: 10.1242/dev.160333

Williamson KA, FitzPatrick DR (2014) The genetic architecture of microphthalmia, anophthalmia and coloboma. European journal of medical genetics 57: 369-80. doi: 10.1016/j.ejmg.2014.05.002

Wisely CE, Sayed JA, Tamez H, Zelinka C, Abdel-Rahman MH, Fischer AJ, Cebulla CM (2017) The chick eye in vision research: An excellent model for the study of ocular disease. Prog Retin Eye Res 61: 72-97. doi: 10.1016/j.preteyeres.2017.06.004

\section{Figure Legend}

Figure 1. Tissue regulatory interactions in the chicken optic cup. The scheme represents inductive and regulatory interactions among eye tissues that have been characterized during optic cup development in the chicken. The main eye territories (Neural retina, Nr; Retinal pigmented epithelium, Rpe; Lens vesicle, Lv; and Periocular mesenchyme, Pom) and the different tissue interactions are colour-coded. The few regulators of relevant tissues' interactions, either signalling molecules or transcription factors, discussed in the text are indicated in the appropriate coloured box. 


\section{Bovolenta and Martinez-Morales Figure 1}

\section{Tissue regulatory interactions in the chicken optic cup}

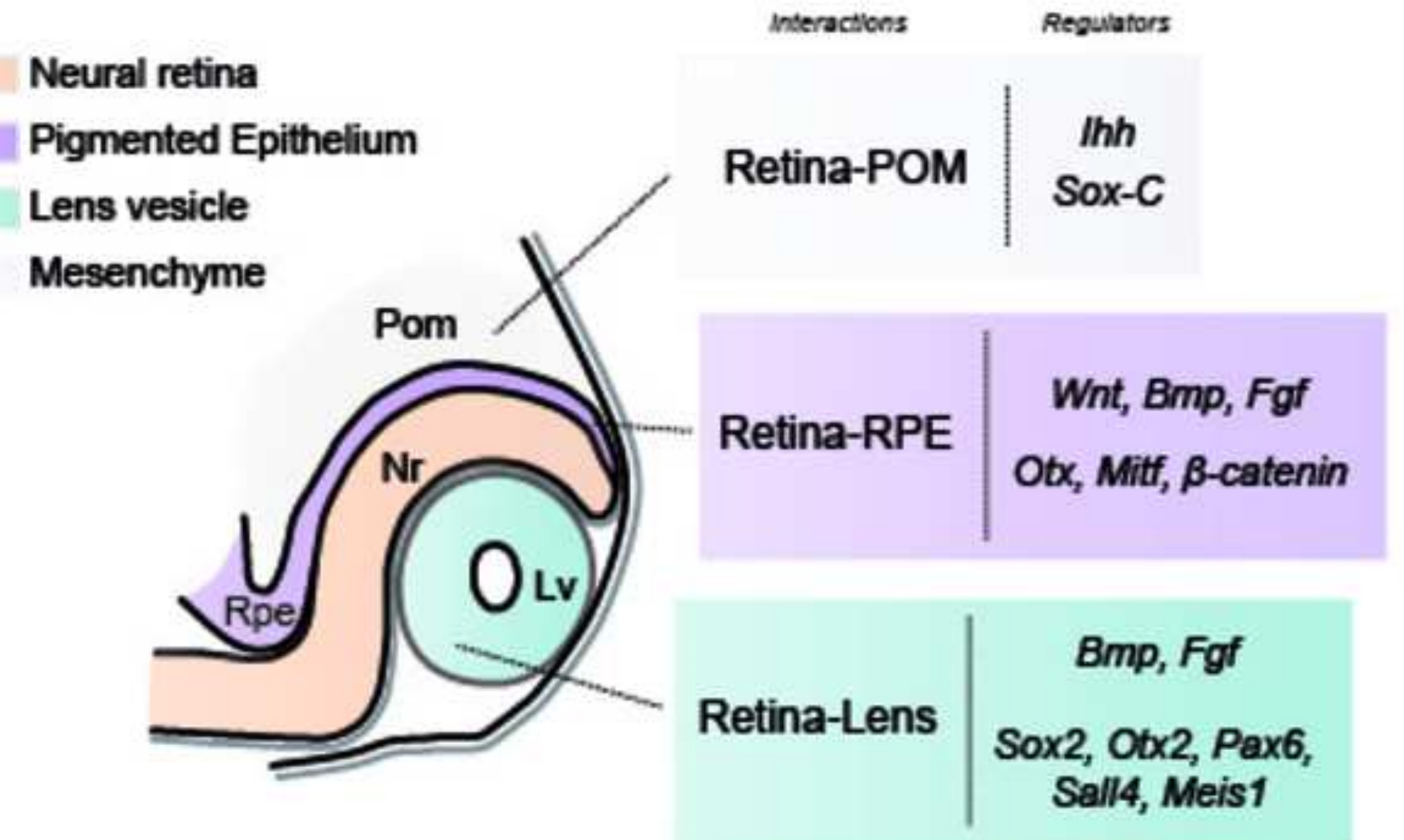

Interactions Regulators

retina

Pigmented Epithelium

Lens vesicle

Mesenchyme

Otx, Mitf, $\beta$-catenin

Sall4, Meis1 